\title{
Adaptive Impedance Control to Enhance Human Skill on a Haptic Interface System
}

\author{
Satoshi Suzuki and Katsuhisa Furuta \\ Department of Robotics and Mechatronics, School of Science and Technology for Future Life, Tokyo Denki University, 5 Asahi-Chou, \\ Senju, Adachi-Ku, Tokyo 120-8551, Japan \\ Correspondence should be addressed to Satoshi Suzuki, ssuzuki@fr.dendai.ac.jp
}

Received 5 December 2011; Revised 9 March 2012; Accepted 30 March 2012

Academic Editor: Lili Ma

Copyright ( $\odot 2012$ S. Suzuki and K. Furuta. This is an open access article distributed under the Creative Commons Attribution License, which permits unrestricted use, distribution, and reproduction in any medium, provided the original work is properly cited.

\begin{abstract}
Adaptive assistive control for a haptic interface system is proposed in the present paper. The assistive control system consists of three subsystems: a servo controller to match the response of the controlled machine to the virtual model, an online identifier of the operator's control characteristics, and a variable dynamics control using adaptive mechanism. The adaptive mechanism tunes an impedance of the virtual model for the haptic device according to the identified operator's characteristics so as to enhance the operator's control performance. The adaptive law is derived by utilizing a Lyapunov candidate function. Using a haptic interface device composed by a $x y$-stage, an effectiveness of the proposed control method was evaluated experimentally. As a result, it was confirmed that the operator's characteristics can be estimated sufficiently and that performance of the operation was enhanced by the variable dynamics assistive control.
\end{abstract}

\section{Introduction}

An impedance control is a key technology of the force/ motion control for any mechanical systems such as an active vehicle suspension, a power steering system, a machining and handling by manipulators, and a tele-operation system. Since dynamics of such controlled mechanism can be adjusted by changing the virtual impedance model, this method is effective to adapt to an ever-changing environment and conditions. Also a biological system has acquired similar strategy of the variable impedance control in the course of an evolution. It is known that an impedance of a musculoskeletal system is changed dynamically during walking, running, and moving the hand [1]. Therefore, variable impedance methods have been studied for artificial legs/orthosis [2] and material-handling machines [3, 4]. Parameters of those impedance control methods are, however, often tuned empirically and intuitively; hence, the system designers have to adjust them according to individuals. Due to this issue, users sometimes have to adapt themselves to the controlled machine when the tuning condition given by the designer is not adequate for the user.
To resolve this paradox, the following approach is ideal; the control characteristics of each user are identified during the operation, and then control of the machine is adjusted adaptively according to the identified user's characteristics. While several similar approaches concerning online variable impedance control are reported, troublesome processes such as a training phase [5] or an empirical tuning for different types of motion [6] are required. Since these approaches are not real adaptive control, a realization of an automatic tuning mechanism without intervention of system designers is expected. Therefore, the present paper presents a design procedure of true adaptive variable impedance control for an assisting system which is considered with the following properties based on the previous method presented in [7]:

(a) adaptivity to control characteristics of individual user,

(b) derivation of adaptive law for variable impedance tuning based on an adaptive control theory.

Main purpose of the control design proposed here is a development of an adaptive tuning law of the machine dynamics, 
of which parameters are fixed in an ordinary mechatronics system, in order to enhance manipulation performance of whole of a human-machine system. And, the purposes of the present study are as follows:

(c) experimental evaluation,

(d) confirmation of the benefit and issue.

Item (a) is realized with an on-line identification based on an assumed model of the human controller. Concerning item (b), an adaptive control law to adjust impedance parameters of the virtual model is derived to ensure the stability and performance of the whole human-machine system. Item (c), evaluation, was performed using a haptic interface device through a point-to-point operation task. Issues and analysis denoted at item (d) are discussed based on the results of the experiment.

This paper is organized as follows. In Section 2, a concept of the adaptive impedance control and its background are mentioned. The haptic interface system and task which were used in the experiment are explained there. Section 3 explains a procedure of the presented assistive control, and its theoretical proof is given there. Section 4 shows results of the experiment and analysis to confirm the effectiveness of the presented method. Last Section 5 presents the conclusion.

\section{Human Assistive System}

2.1. Concept of Adaptive Impedance Control. In order to design an assistive mechanism for user's manipulation in a human-in-the-loop system, an adequate human modeling and a feasible assistive control are required. Human modeling has been studied in the field of control engineering from its early beginnings such as a linear servo control model [8], a PID-base time-variant model [9], and an optimal control model [10]. Those models can express a human behavior well for each assumed situation; however, it is inadequate to explain the learning process of a user from the beginner phase to the expert phase. To find adequate model which can treat a human adaptability, it is adequate to refer the voluntary motion control of a musculoskeletal system. The reason is that such model is formulated to explain a process of a human development, and most popular model is a feedback-error-learning model [11, 12]. This model can be utilized to explain the learning of an external unknown dynamics since such an external system can be thought as an extension of our body. On the learning process of the external dynamics, a delay has to be considered because it concerns the stability and performance of whole human-machine system. The delay arises certainly at the visual processing and at the neural transmission between a brain and sensory receptor/muscles. Such undesirable effect given by the delay is compensated by an internal feedback compensation using an efferent neuron and by a delay compensation mechanism which is explained by the Smith predictor theory [13]. Additionally, as shown in Kleinman's research of the dynamics of a pilot [10], a human (i.e., pilot) has a high ability to compensate the delay in the response of a vehicle. Hence, if a time-delay effect inside the

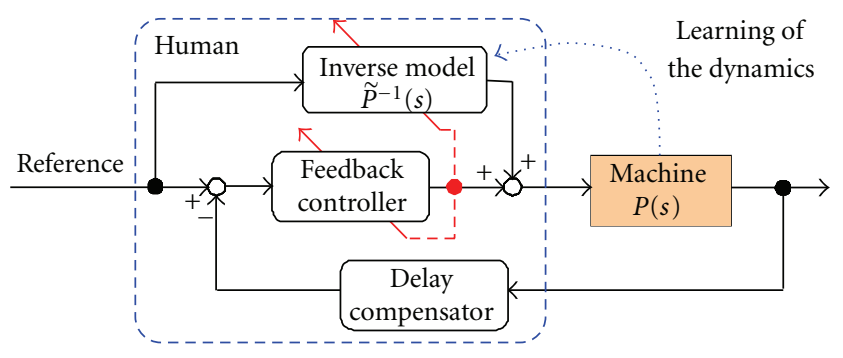

FIGURE 1: Human model inside a human-machine system.

machine side system does not change, it is expected that the delay in the human-machine system can be compensated relatively easily. Therefore, the block diagram shown in Figure 1 appears adequate for designing of a human-machine assist system. The model mentions that human learns the unknown dynamics of the machine and uses the identified model as an inverse model for the manipulation of the machine.

The concept shown in Figure 1 indicates that difference of the machine's dynamics affects indirectly to the learning of the operator. If the operated machine can change its own dynamics characteristics so as to be learned by individual operator without difficulty, performance of the operation would be enhanced. Therefore, in order to enhance the operator's performance, as shown in Figure 2, an original dynamics of the machine is replaced to a virtual dynamics model from the operator's side by making a local loop feedback with a virtual internal model control. In short, the impedance of the virtual dynamics model is modified so as to decrease an error which relates with each task performance. To summarize this discussion, the following three functions are required to realize aforementioned adaptive impedance control.

Step 1. Virtual internal model (VIM) control.

Step 2. Online identification of the operator.

Step 3. Adaptive mechanism to tune the VIM.

The VIM control for Step 1 is realized by making a local servo system that tracks the output of a virtual impedance model. The servo control input law is designed using Linear-Quadratic Regulator (LQR). Identification for Step 2 is performed by assuming a parametric model of operator's control characteristics. Concerning Step 3, the adaptive mechanism is designed by changing the impedance parameters of the VIM obtained at the Step 3 after derivation of the adaptive law of the VIM model based on a Lyapunov-like function. Details of this process are explained in Section 3.

2.2. Experimental System. A haptic interface system, which is shown in Figure 3, was used to evaluate the adaptive assistive control presented in Section 3. The haptic device consists of a two degree-of-freedom planer $x y$-stage, produced by NSK corporation, and a real-time CG monitor programmed by visual $\mathrm{C}++$. The $x y$-stage is driven by two linear direct drive 


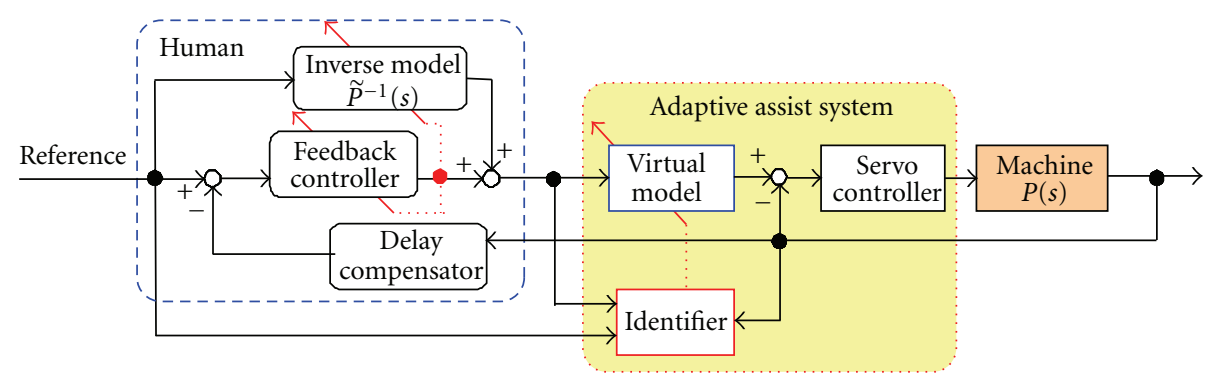

FIgURE 2: Structure of human assistive system with the adaptive impedance control.

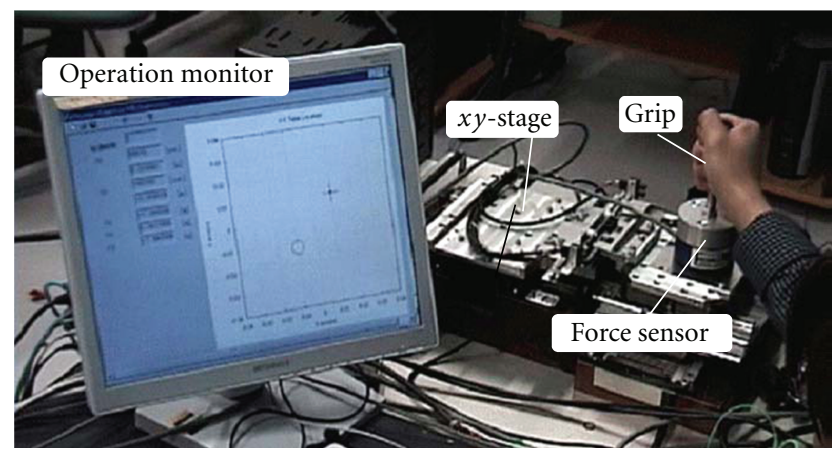

FIGURE 3: Haptic interface devices.

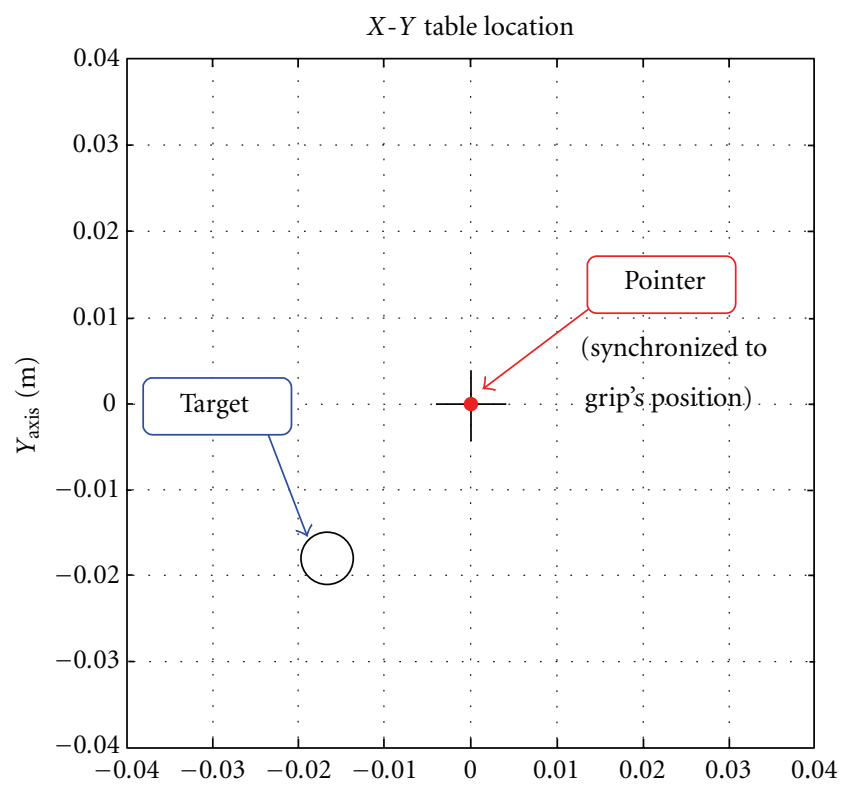

FIGURE 4: Operation monitor of the haptic interface system.

motors. The operator moves a grip attached to the $x y$-stage and a pointer displayed on the CG monitor (see Figure 4) is also moved according to the position of the grip. Operator's hand force is measured by a 6-axis force sensor embedded between the grip and the stage. The $x$ - and $y$-axes of the stage do not effect each other because of a mechanically independent design. Computations of the control of the stage and the CG displaying are executed by a PC/AT $3 \mathrm{GHz}$ computer under real-time scheduling control. The control interval is $2 \mathrm{~ms}$, and the movable range is about $62 \mathrm{~mm}$ in both $x$ and $y$ directions.

2.3. Task of Manipulation. Since a point-to-point (PTP) manipulation is a popular task both in a daily life and in an industrial situation, the PTP-task was adopted for verification of the presented method. The PTP tasks were repeated by changing the target's position at random so as to keep the distance constant from each last target to the next one. As soon as the target is displayed on the monitor, the operator moves the pointer to the target by manipulating the grip of the $x y$-stage. When position of the pointer is kept inside the target circle for 3 seconds, one PTP motion (one trial) is finished, and then a new next target circle is displayed at random. To reduce the fatigue of the participant, ten-second rest was given to the participant after every five trials.

\section{Design of Adaptive Impedance Control}

3.1. Virtual Internal Model Control (Step 1). A procedure to apply the virtual internal model (VIM) control [14] to the $x y$-stage is mentioned in this section. Any mechanical mechanism includes nonlinearity caused by friction, variances of viscosity, and unknown dynamics; hence, it is difficult to apply a linear system control theory to actual machine without any nonlinear compensation. Since VIM is effective to suppress inherent characteristics of mechanical components such as frictions, an adaptive control for linear systems can produce an effect. Although the haptic system used in the experiment has two degree-of-freedom motions, controllers of the $x$ - and $y$-axes can be designed separately thanks to the mechanical independence; hence, subscripts of $x$ and $y$ are omitted in later explanation. Variables and parameters of the haptic device model are shown in Figure 5 and Table 1.

The block diagram of the virtual internal model control is shown in Figure 6 and the related variables and parameters are summarized in Table 2. Dynamic equations of the stage and the virtual model are expressed as follows:

$$
\begin{gathered}
m_{p} \ddot{x}_{p}+d_{p} \dot{x}_{p}=f_{h}+f_{a}, \\
m_{r} \ddot{x}_{r}+d_{r} \dot{x}_{r}=f_{h} .
\end{gathered}
$$




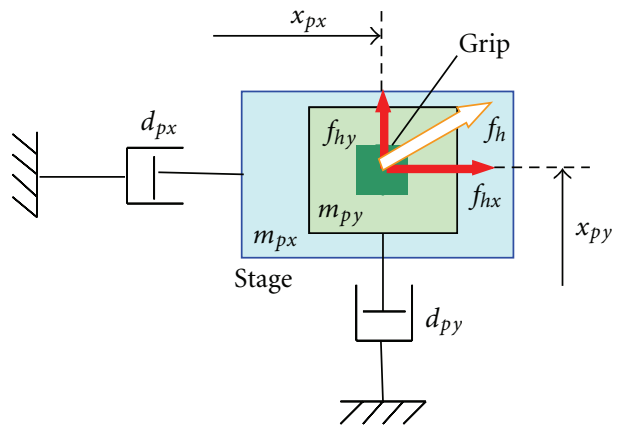

FIGURE 5: Model of the $x y$-stage.

TABLE 1: Parameters and variables of haptic interface device.

\begin{tabular}{lcl}
\hline Variables/parameters & Unit & Meanings \\
\hline$x_{p *}$ & {$[\mathrm{~m}]$} & Position of a grip \\
$f_{h *}$ & {$[\mathrm{~N}]$} & Force from an operator \\
$f_{a *}$ & {$[\mathrm{~N}]$} & Force from an actuator \\
$m_{p *}$ & {$[\mathrm{~kg}]$} & Mass of a stage \\
$d_{p *}$ & {$[\mathrm{Ns} / \mathrm{m}]$} & Viscosity of a stage \\
\hline
\end{tabular}

$*: x$ or $y$ for $x$ - and $y$-axis.

TABLE 2: Parameters and variables for virtual model control.

\begin{tabular}{lcl}
\hline Variables/parameters & Unit & Meanings \\
\hline$x_{r}$ & {$[\mathrm{~m}]$} & Position of a virtual model \\
$e_{r}$ & {$[\mathrm{~m}]$} & Error $\left(=: x_{r}-x_{p}\right)$ \\
$m_{r}$ & {$[\mathrm{~kg}]$} & Mass of a virtual model \\
$d_{r}$ & {$[\mathrm{Ns} / \mathrm{m}]$} & Viscosity of a virtual model \\
\hline
\end{tabular}

Defining an error as $e_{r}:=x_{r}-x_{p},(1)$ and (2) are transformed into

$$
\begin{gathered}
\frac{d}{d t}\left[\begin{array}{c}
e_{r} \\
\dot{e}_{r}
\end{array}\right]=\left[\begin{array}{ll}
0 & 1 \\
0 & 0
\end{array}\right]\left[\begin{array}{c}
e_{r} \\
\dot{e}_{r}
\end{array}\right]+\left[\begin{array}{l}
0 \\
1
\end{array}\right] u \\
u:=\frac{-d_{r} \dot{x}_{r}+f_{h}}{m_{r}}+\frac{d_{p} \dot{x}_{p}-f_{h}-f_{a}}{m_{p}} .
\end{gathered}
$$

Minimizing the error defined by (3) makes the stage conform to a response of the virtual model described by (2). To compensate steady-state error, the integral variable $\int e_{r}$ is taken into consideration in the state vector as follows:

$$
\frac{d}{d t} z=A z+B u
$$

where

$$
A:=\left[\begin{array}{ccc}
0 & 1 & 0 \\
0 & 0 & 1 \\
0 & 0 & 0
\end{array}\right], \quad B:=\left[\begin{array}{c}
0 \\
0 \\
1
\end{array}\right], \quad z:=\left[\begin{array}{c}
\int e_{r} \\
e_{r} \\
\dot{e}_{r}
\end{array}\right] .
$$

The control law is calculated using an LQR method with the quadratic criterion:

$$
J=\int_{0}^{\infty}\left(z^{T} Q z+u^{T} R u\right) d t
$$

where a positive semi-definite $Q \in R^{3 \times 3}$ and a positive definite $R \in R^{1 \times 1}$ are weighting matrices. The input is given as

$$
u=-F z, \quad F:=R^{-1} B^{T} P
$$

where $P$ is a symmetry positive-definite matrix of a Riccati Algebraic Equation given by

$$
P A+A^{T} P-P B R^{-1} B^{T} P+Q=0 .
$$

Since (7) is expanded as

$$
-F z=\frac{-d_{r} \dot{x}_{r}+f_{h}}{m_{r}}+\frac{d_{p} \dot{x}_{p}-f_{h}-f_{a}}{m_{p}},
$$

a final form of the control law is obtained as follows:

$$
f_{a}=\frac{m_{p}}{m_{r}}\left(f_{h}-d_{r} \dot{x}_{r}\right)+d_{p} \dot{x}_{p}-f_{h}-m_{p} F z
$$

For the actual apparatus used in the experiment, parameters of VIM were specified as $m_{r x}=m_{r y}=50[\mathrm{~kg}]$ and $d_{r x}=$ $d_{r y}=50[\mathrm{Ns} / \mathrm{m}]$ to intentionally obtain a slightly difficult manipulation feeling as a training test with consideration of input range of the actuators. The weighting matrices in (6) for the LQR servo design were decided as $Q=$ $\operatorname{diag}\left(150,1.12 \times 10^{7}, 5100\right)$ and $R=1$. As a result, the feedback gain matrix was obtained as $F=[10.9,3007.9,100.7]$.

3.2. Online Identification of Human Control Characteristics (Step 2). Human control characteristics are complex because various kinds of compensators, such as an oculomotor control, a proprioceptive control, and a neuromuscular control, are related to each other [13]. There is, however, a fairly large body of data that can be explained by a linear model plus time delay [15] when an operation condition is limited. One of most famous models supporting such linear model assumption is a crossover model. This model insists that a frequency transfer function of a skilled operator in a man-machine system adapts to make the total system kept unchanged under a variation of the controlled system dynamics. In other words, human changes own control characteristics so that a closed-loop transfer function of whole human-machine system becomes a first-order system at a wide frequency band and the human plays a role of simple linear model. Also in previous study of the present authors, an identification analysis of the skilled operator's frequency characteristics showed an existence of the cross-over model through a juggling task using a haptic test device [16]. Hence, whole system relating a voluntary motion is simplified into the three components in this study: a linear controller inside a brain, a neuromuscular dynamics, and reaction time delay. After a learning of the machine dynamics is sufficiently finished, the human can be considered as a simple feedback controller which moves the grip to the target position by watching the monitor in case of the PTP task. Finally, a block diagram of a visual voluntary motion control is assumed as a feedback model as shown in Figure 8. In the figure, $r$ is a reference position for a pointer, $e_{h}$ is an error between the 


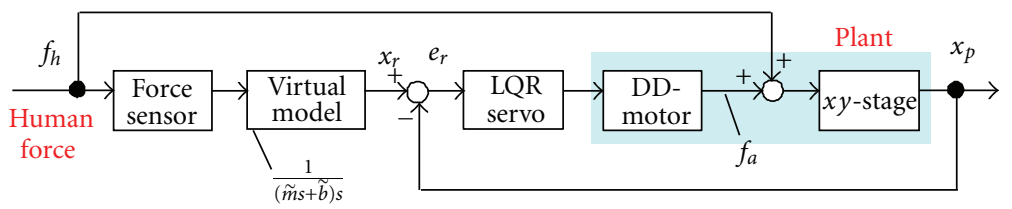

FIGURE 6: Block diagram of virtual internal model control.

target and the present pointer, and $u_{h}$ is an input computed by a brain controller. Here, the plant block is a virtual $x y$-stage of which impedance property is adjusted by the VIM control. The neuromuscular dynamics can often be approximated by a first-order lag [17] and a simplest human controller is a PD controller [9]; hence, the human transfer function, $G_{h}^{\prime}(s)$, is assumed in this study as

$$
G_{h}^{\prime}(s)=\frac{K_{d} s+K_{p}}{T s+1} e^{-L s},
$$

where $K_{p}, K_{d}, T$, and $L$ are a proportional gain of the human brain controller, the differential gain of it, a time constant of the neuromuscular system, and reaction time delay, respectively. As discussed in Section 2.1, compensation of the delay factor is necessary not only for voluntary motion control in a human but also for an adequate human-machine system, and a human has an excellent ability to compensate the delay effect. And an influence of the delay to the control characteristics of the whole human-machine system depends on a response speed of the machine and the task condition. Therefore, in the present study, the response delay of participants was investigated as a preliminary experiment using the VIM control which was designed at Section 3.1. Participant aged 22 years was requested to execute the PTP manipulation hundred times. The time that the pointer begins moving just after the new target circle was displayed on the monitor was counted as the response delay. Figure 7 shows the change of the measured time delay. The dots represent measured values, and the solid lines express an approximated thirdorder polynomial fitting curve from the measured data. This graph shows no conclusive relationship between time delay and the number of trials, and the value is almost constant at about 0.4 second. Additional nine participants showed same tendency, and significant difference between individuals was not confirmed. For this reason, it was expected that the simple data shift would be sufficient to compensate the delay effect in the identification for the present study. Therefore, the time delay factor described in (11) was omitted for the identification by shifting the measured data for the 0.4 second as a rest time, and the following model was considered for later process:

$$
G_{h}(s)=\frac{K_{d} s+K_{p}}{T s+1} .
$$

Applying a bilinear transformation

$$
s \simeq \frac{2}{\Delta} \cdot \frac{1-z^{-1}}{1+z^{-1}}
$$

to (12) yields the following discrete impulse transfer function $G_{h}[z]$ :

$$
\begin{aligned}
G_{h}[z] & =\frac{b_{1} z^{-1}+b_{0}}{a_{1} z^{-1}+1}, \\
a_{1} & :=\frac{-2 T+\Delta}{2 T+\Delta}, \\
b_{0} & :=\frac{2 K_{d}+K_{p} \Delta}{2 T+\Delta}, \\
b_{1} & :=\frac{K_{p} \Delta-2 K_{d}}{2 T+\Delta},
\end{aligned}
$$

where $\Delta$ is a sampling interval. From (15)-(17), following equations are derived:

$$
\begin{gathered}
T=\frac{\Delta}{2} \frac{1-a_{1}}{1+a_{1}}, \\
K_{p}=\frac{2 T+\Delta}{2 \Delta}\left(b_{0}+b_{1}\right), \\
K_{d}=\frac{2 T+\Delta}{4}\left(b_{0}-b_{1}\right) .
\end{gathered}
$$

If $a_{0}, b_{0}$, and $b_{1}$ are identified from the input/output response data, characteristic parameters of the human controller can be derived using (18). These parameters are used in a design of the next variable dynamics assistive controller.

3.3. Variable Dynamics Assist Control (Step 3). An assistive control proposed in this paper changes dynamics of the internal model on-line depending on operator's characteristics. A block diagram of the assistive control is shown in Figure 9(a). In the figure, $r, y, e, v$, and $f$ are a positional reference, a position of the stage, the error, an output of a brain controller, and a force generated by the hand, respectively. It is assumed that (a) $K_{p}, K_{d}$, and $T$ are time-slowing changing parameters and that (b) parameters of a virtual machine $\widetilde{m}$ and $\tilde{b}$ can be tuned, because the assumption (b) is realized by the VIM control, that is, $\tilde{m}$ and $\tilde{b}$ are adjustable parameters in this scheme. Figure 9(a) expresses a general human-machine system that includes a human controller $\left(K_{p}+K_{d}\right) /(T s+$ 1) $=: C$ and a plant $1 /(\tilde{m} s+\tilde{b}) s=: P$ for virtual haptic interface device. It can be considered conversely that the system consists of a plant $C$ changing slowly the parameters $\left(K_{p}, K_{d}\right.$, and $\left.T\right)$ and the controller $P$ having directly variable coefficients $(\tilde{m}$ and $\tilde{b})$, as shown in Figure $9(\mathrm{~b})$.

Note that the output $y$ of new controller $P$ cannot be changed arbitrarily and that only tuning of the controller's 


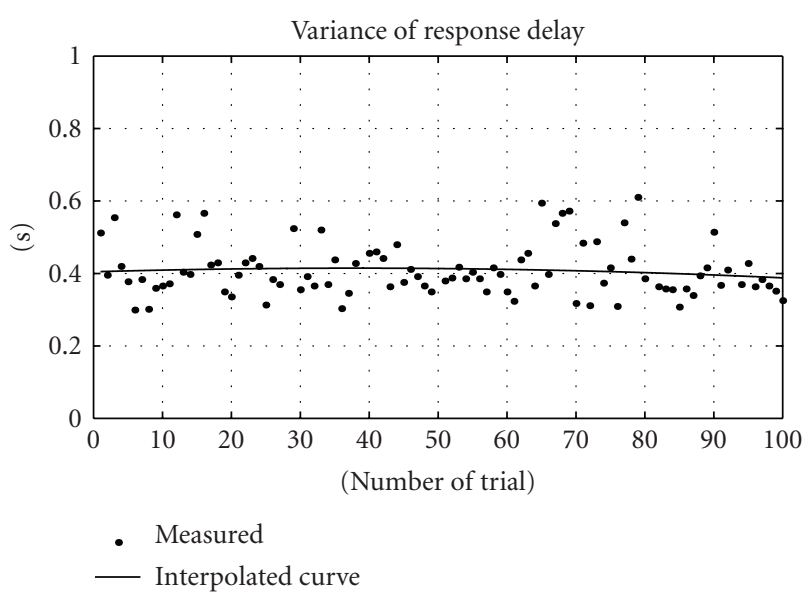

FIGURE 7: Variance of response delay.

coefficients is possible. Moreover, transformation of the block diagram shown in Figure 9(b) yields a general feedback form, as shown in Figure 9(c). In the following, in order to avoid misunderstanding owing to habits, characters for variables $x$ and $u$ are used instead of $f$ and $e$, respectively. Then, the following equations are obtained:

$$
\begin{aligned}
x(s) & =\frac{K_{p}+K_{d} s}{T s+1} u(s), \\
u(s) & =\frac{1}{(\tilde{m} s+\tilde{b}) s} e^{\prime}(s), \\
e^{\prime}(s) & :=r^{\prime}(s)-x(s), \\
r^{\prime}(s) & :=(\tilde{m} s+\tilde{b}) s \cdot r(s) .
\end{aligned}
$$

The purpose of the PTP task is a tracking such that $y \rightarrow r$ in the original block diagram shown in Figure 9(a). This means that $e \rightarrow 0$ (in Figure $9(\mathrm{~b})$ ), that is, $u \rightarrow 0$ (in Figure 9(c)); then (20) indicates that $e^{\prime} \rightarrow 0$ as $t \rightarrow \infty$. First, choosing a Lyapnov candidate $V$ as $V:=(1 / 2) e^{\prime}(t)^{2}$, the condition of convergence is investigated. It can be considered that a closed-loop system shown in Figure 9(c) is almost stable under the assumptions of (a) and (b); hence, it is not always necessary that $d V / d t<0$ holds for keeping the stability. Second, an update law for $\tilde{m}$ and $\tilde{b}$ is derived using a Lyapunov-like analysis. If a step input is chosen for $r(t)$ for the PTP motion, the response of $r^{\prime}(t)$ defined by (22) becomes almost impulse shape. The moment of $t=0$ is, however, not important practically because the purpose of the control is an enhancement of the performance of the motion by making the tracking error small which occurs mainly by the positioning near the target position. In addition, the impulsive response converges into zero rapidly, hence, an approximation as $d r^{\prime}(t) / d t \simeq 0$ holds if $t \gg 0$.
Then, the time-derivative of $V$ can be approximated and can be transformed as follows:

$$
\begin{aligned}
\frac{d}{d t} V(t) & =e^{\prime}(t) \frac{d}{d t} e^{\prime}(t) \simeq-e^{\prime}(t) \frac{d}{d t} x(t) \quad(t>0) \\
& =-e^{\prime}(t) \frac{d}{d t} \mathcal{L}^{-1}\left[\frac{K_{p}+K_{d} s}{T s+1} \frac{1}{(\tilde{m} s+\tilde{b}) s} e^{\prime}(s)\right] \\
& =-e^{\prime}(t) \mathcal{L}^{-1}\left[\frac{K_{p}+K_{d} s}{T s+1} \frac{1}{(\tilde{m} s+\tilde{b})} e^{\prime}(s)\right] \\
& =-e^{\prime}(t) \mathcal{L}^{-1}\left[\frac{K_{p}-K_{d} / T}{\tilde{b} T-\tilde{m}} \cdot \frac{1}{s+1 / T} e^{\prime}(s)\right. \\
\left.+\frac{K_{p}-K_{d} \tilde{b} / \tilde{m}}{\tilde{m}-T \tilde{b}} \cdot \frac{1}{s+\tilde{b} / \tilde{m}} e^{\prime}(s)\right] & \\
& =-e^{\prime}(t)\left\{\begin{array}{c}
\frac{K_{p}-K_{d} / T}{\tilde{b} T-\tilde{m}} \cdot \phi\left(t, \frac{1}{T}\right) \\
\left.+\frac{K_{p}-K_{d} \tilde{b} / \tilde{m}}{\tilde{m}-T \tilde{b}} \cdot \phi\left(t, \frac{\tilde{b}}{\tilde{m}}\right)\right\}
\end{array}\right.
\end{aligned}
$$

where the function $\phi(t, \alpha)$ is defined as

$$
\phi(t, \alpha):=\int_{0}^{t} e^{-\alpha(t-\tau)} \cdot e^{\prime}(\tau) d \tau .
$$

Since it is necessary for each term in (23) to be negative in order to satisfy $d V / d t<0$ as long as possible, the following conditions are considered:

$$
\begin{gathered}
\frac{K_{p}-K_{d} / T}{\tilde{b} T-\tilde{m}}>(<) 0 \quad \text { if } e^{\prime}(t) \phi\left(t, \frac{1}{T}\right)>(<) 0, \\
\frac{K_{p}-K_{d} \tilde{b} / \tilde{m}}{\tilde{m}-T \tilde{b}}>(<) 0 \quad \text { if } e^{\prime}(t) \phi\left(t, \frac{\tilde{b}}{\tilde{m}}\right)>(<) 0 .
\end{gathered}
$$

Conversely, if parameters do not fulfill the previous inequality conditions, variable parameters $\tilde{m}$ and $\tilde{b}$ are tuned so as the unsatisfied condition will be recovered. Now, the following intermediate variables are introduced:

$$
\begin{aligned}
& \delta_{1}:=\eta_{1} \cdot(\tilde{b} T-\tilde{m}), \\
& \delta_{2}:=\eta_{2} \cdot(\tilde{m}-T \tilde{b}), \\
& \eta_{1}:=\operatorname{sgn}\left(K_{p}-\frac{K_{d}}{T}\right) \cdot \operatorname{sgn}\left\{e^{\prime}(t) \phi\left(t, \frac{1}{T}\right)\right\}, \\
& \eta_{2}:=\operatorname{sgn}\left(K_{p}-\frac{K_{d} \tilde{b}}{\tilde{m}}\right) \cdot \operatorname{sgn}\left\{e^{\prime}(t) \phi\left(t, \frac{\tilde{b}}{\tilde{m}}\right)\right\} .
\end{aligned}
$$

By checking signs of a numerator and a denominator of (25) and signs of $\tilde{m}$ and $\tilde{b}$, the following update law can be considered:

$$
\begin{gathered}
\tilde{b}[t+\Delta] \longleftarrow \tilde{b}[t]+k_{1} \sigma\left(\delta_{1}\right) \eta_{1} \cdot|e|, \\
\tilde{m}[t+\Delta] \longleftarrow \tilde{m}[t]-k_{2} \sigma\left(\delta_{1}\right) \eta_{1} \cdot|e|,
\end{gathered}
$$




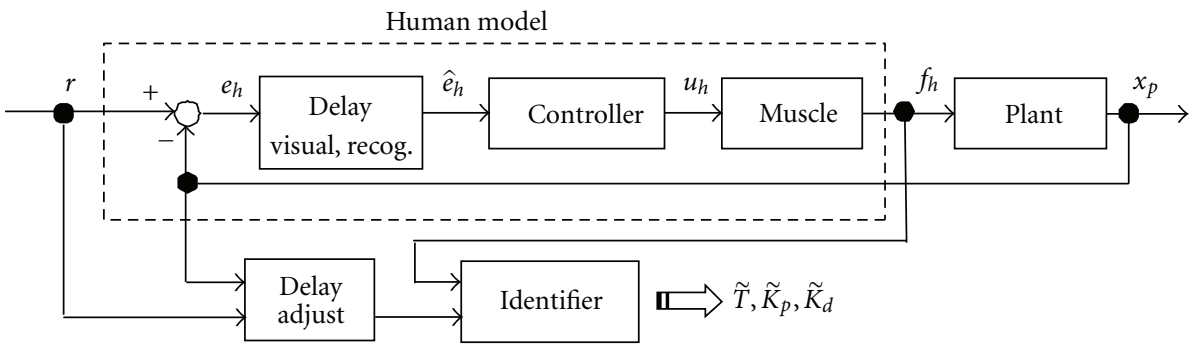

Figure 8: A human control model and its identification.

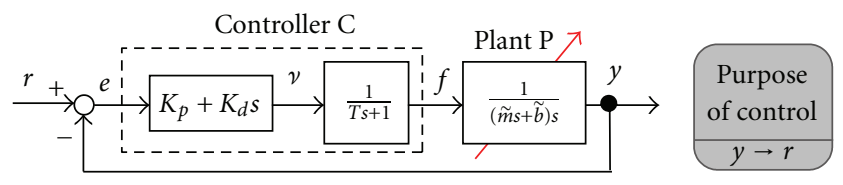

(a)

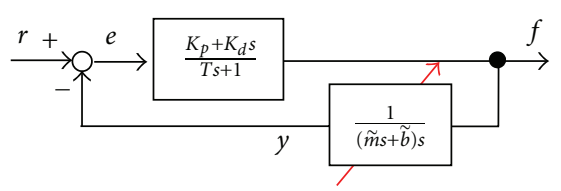

(b)

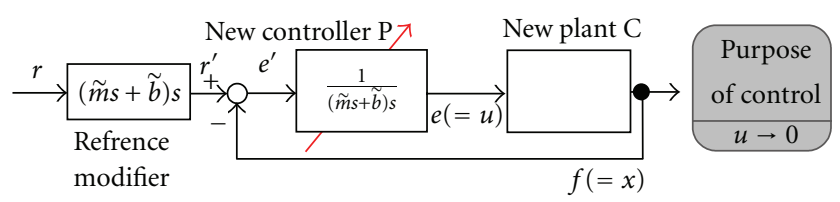

(c)

FIGURE 9: Transformation of block diagrams for variable dynamics control.

where $k_{1}$ and $k_{2}$ are positive constant parameters, $\Delta$ is a control interval, brackets in previous equations mean a discrete-time point, and a function $\sigma$ is defined as

$$
\sigma(\delta)= \begin{cases}0, & \delta>0 \\ |\delta|, & \delta<0\end{cases}
$$

The other update law is derived from (26) in same manner as follows:

$$
\begin{aligned}
& \tilde{b}[t+\Delta] \longleftarrow \tilde{b}[t]-k_{3} \sigma\left(\delta_{2}\right) \eta_{2} \cdot|e|, \\
& \tilde{m}[t+\Delta] \longleftarrow \tilde{m}[t]+k_{4} \sigma\left(\delta_{2}\right) \eta_{2} \cdot|e|,
\end{aligned}
$$

where $k_{3}$ and $k_{4}$ are positive constants. Equations (28)-(30) are summarized into the following parameter update law.

$$
\left[\begin{array}{c}
\tilde{b} \\
\tilde{m}
\end{array}\right]_{[t+\Delta]}=\left[\begin{array}{c}
\tilde{b} \\
\tilde{m}
\end{array}\right]_{[t]}+\left[\begin{array}{cc}
k_{1} & -k_{3} \\
-k_{2} & k_{4}
\end{array}\right]\left[\begin{array}{l}
\sigma\left(\delta_{1}\right) \eta_{1} \\
\sigma\left(\delta_{2}\right) \eta_{2}
\end{array}\right] .
$$

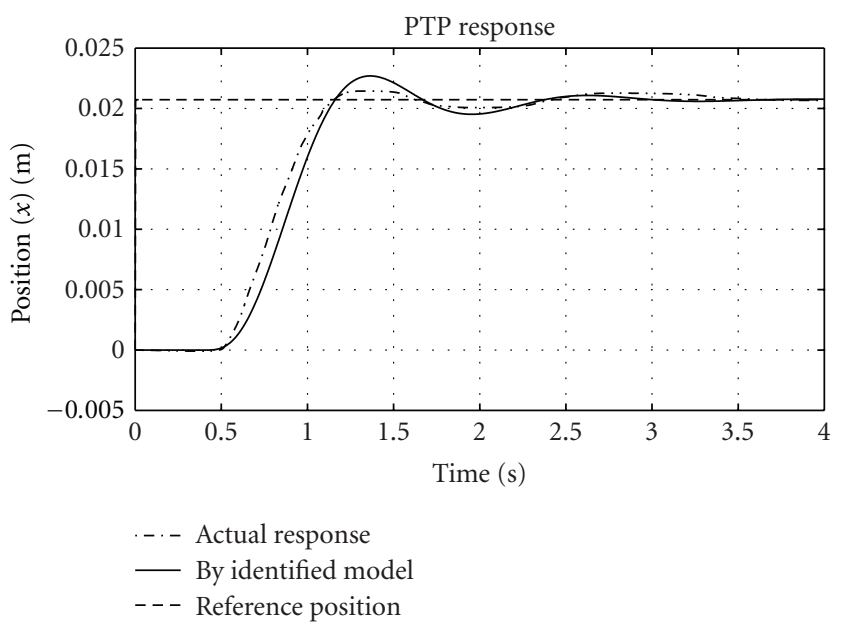

FIGURE 10: Comparison of PTP responses.

On the implementation, these parameters are updated under the following practical limit to avoid an input saturation of actual actuators:

$$
\underline{b}<\tilde{b}<\bar{b}, \quad \underline{m}<\tilde{m}<\bar{m},
$$

where $\underline{b}, \bar{b}, \underline{m}$, and $\bar{m}$ are constant. Here parameters $k_{i}$ are chosen as they satisfy $k_{1} k_{4}-k_{2} k_{3} \neq 0$. Integral computation described in (24) is executed by using the following alternative online recursive computation:

$$
\phi[t, \alpha]=e^{-\alpha \Delta} \phi[t-\Delta, \alpha]+e^{\prime}[t] \Delta .
$$

Since (22) cannot be computed directly, an approximation as $(\tilde{m} s+\tilde{b}) s \simeq(\tilde{m} s+\tilde{b}) s /(0.01 s+1)^{2}$ is used, and the response is computed by the Eular integration with the state-space model which is derived with a controllable canonical form. $K_{p}, K_{d}$, and $T$ are identified on every PTP motion and are updated according to an appropriateness of the identification result.

\section{Experimental Result and Analysis}

4.1. Online Identification of Human Controller. For a design of the VIM control of the $x y$-stage, the initial parameters were chosen as $\tilde{m}[0]=50[\mathrm{~kg}]$, and $\tilde{b}[0]=50[\mathrm{Ns} / \mathrm{m}]$. Input information for the identification was chosen as an error 


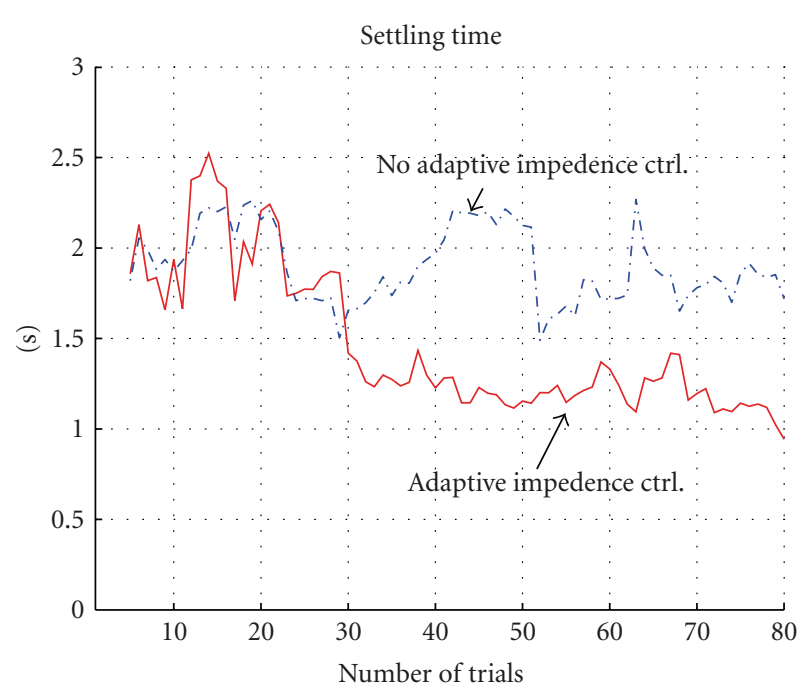

Figure 11: Evolution of settling time.

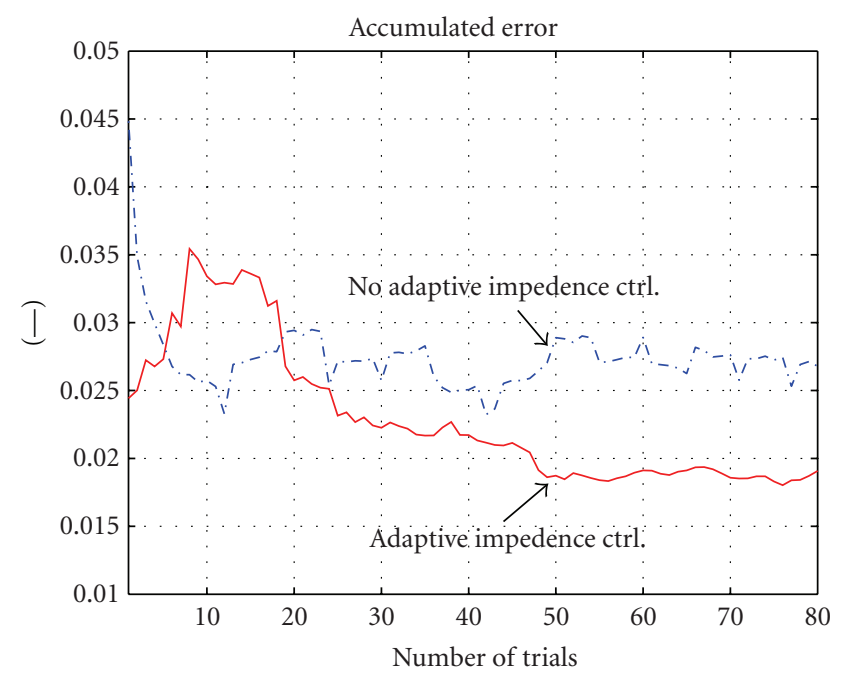

FIgURE 12: Evolution of accumulated error.

between the current position and the target one. Measurement value of force filtered through a $36 \mathrm{~Hz}$ LPF was used as output information for the identification. The time-delay effect was compensated by sifting the measured input signal at every PTP motion. Measured data was decimated by a factor of 10 for an identification; in short, an identification sampling time is $20 \mathrm{~ms}$ to suppress oscillation in the identified parameters. One result of the identification is shown in Figure 10. The solid curve is a simulated step response that was computed using an identified human controller model and virtual dynamics model of the stage. Those identified parameters were $\widehat{K}_{p}=779.0, \widehat{K}_{d}=288.0$, and $\widehat{T}=0.18$, and the time delay was treated as $\widehat{L}=0.406$ in the simulation. Since the response of the identified model resembles to the actual response, it can confirmed that the identification process was reasonable.

4.2. Verification of Assistive Effect. Since a key point of the proposed assistive method is to increase performance of the

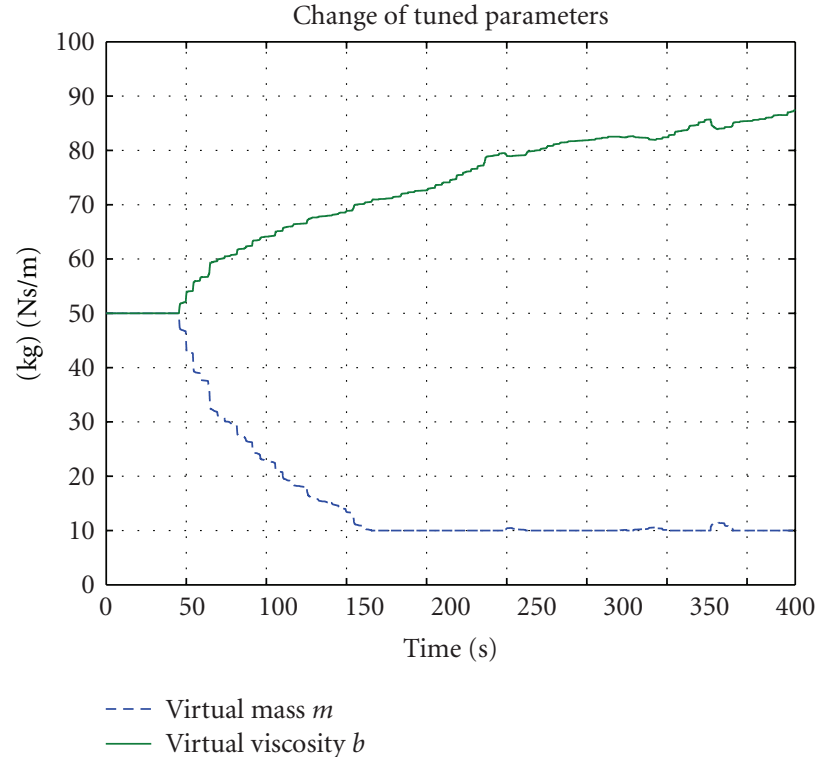

Figure 13: Evolution of tuned parameters.

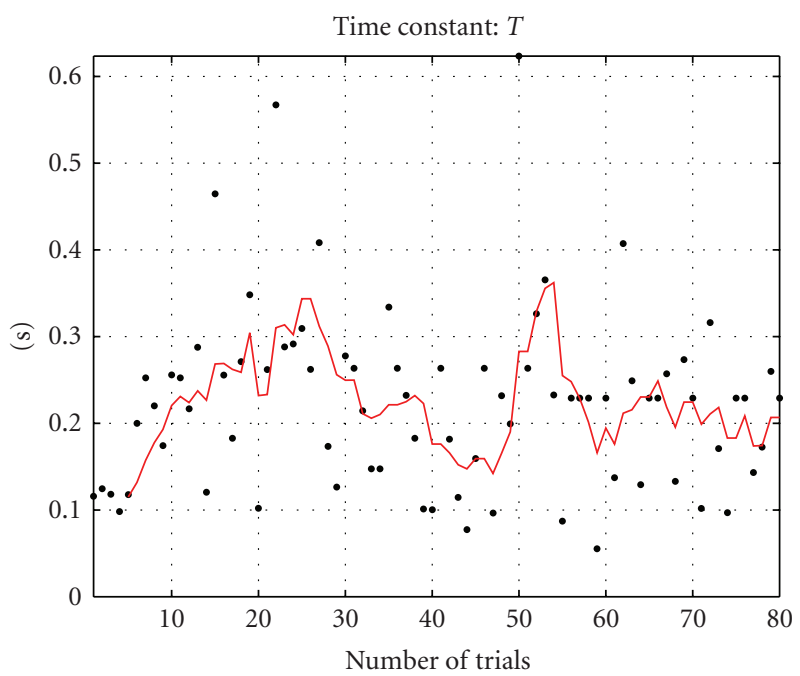

FIgure 14: Change of identified T.

operator's manipulation by adjusting the machine dynamics, we investigated whether the performance of an operator who was used to the PTP operation without the adaptive control could be increased with the proposed adaptive control. From this aim, before the presented assistive control was applied to a participant, sufficient training was given to become a skilled operator using the haptic device tuned with fixed parameters which were same initial values on the assistive control. As a result of this preliminary training, it was confirmed that the performance of the participant became good and did not indicate no further improvement by checking the settling time on the PTP operation.

For the assistive control, parameters of the update law in (31) were chosen as $k_{1}=k_{4}=1 \times 10^{-4}$, and $k_{2}=k_{3}=$ $2 \times 10^{-4}$. Since even the expert showed perturbation in the performance at the beginning of several trials, the normal VIM control was executed from the first trial and the adaptive 


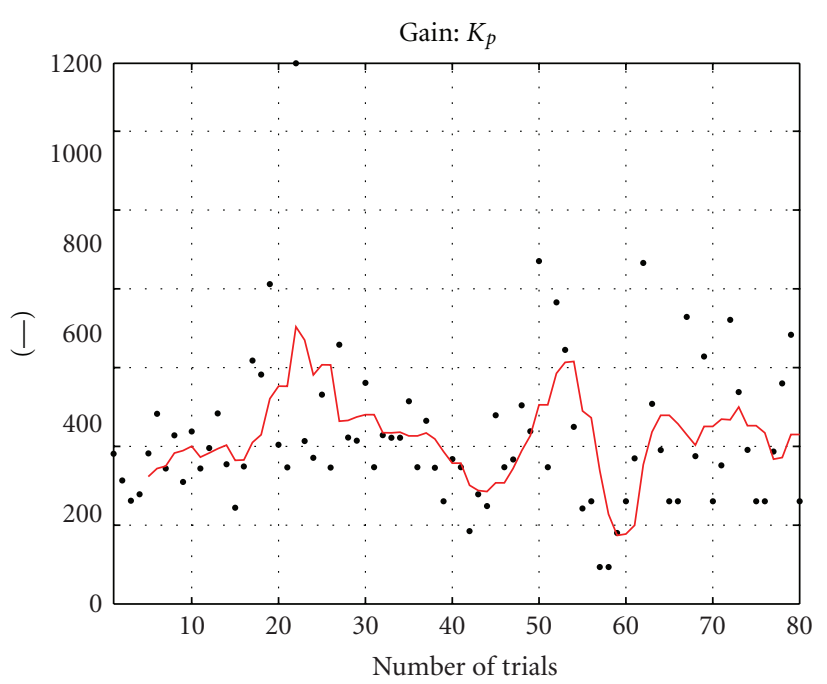

Figure 15: Change of identified $K_{p}$.

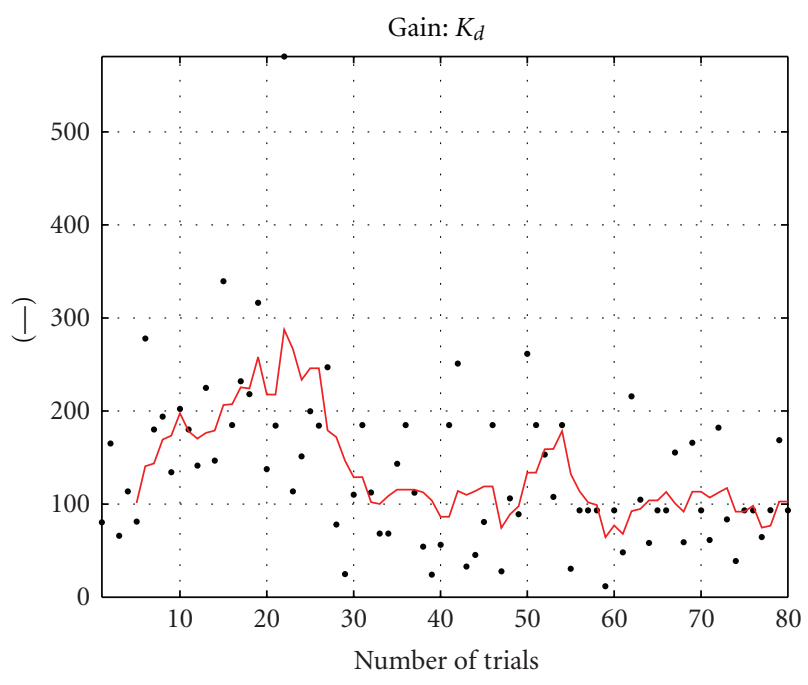

Figure 16: Change of identified $K_{d}$.

impedance control was activated after 50 seconds (about 10 trials). Figure 11 shows an evolution of the settling time from when the new target was displayed on the monitor till when the pointer was reached into the target circle. When three seconds passed after the pointer was kept staying inside the target circle on the monitor, it was judged that the pointer had been moved to the target by the operation. Values of the $y$-axis in the figure show the settling time that does not include three seconds. The solid line shows the result of the adaptive impedance control, and the dotted line shows the other result obtained by nonadaptive impedance control before the participant did not yet try the adaptive impedance control. Each line shows the evolution of trend computed by the moving average computation against five PTP tasks. While the nonadaptive impedance control case shows roughly steady state of 1.8 seconds after 50 trials, the other adaptive impedance control case shows a decrease to about 1.1 seconds. In short, speed of the PTP motion was improved by the adaptive impedance control.

Figure 12 shows an evolution of the accumulation errors $\int|e(t)| d t$ till each settling time at each trial. Similarly, the solid and dotted lines show the results of the adaptive impedance control and nonadaptive control, respectively. It can be confirmed that the accumulation error was also decreasing in case of the adaptive control. Both Figures 12 and 11 demonstrated effects of the presented method.

Figure 13 shows change of tuned parameters $\tilde{m}$ and $\tilde{b}$. At the beginning of trials, these values were constant because the assistive control was activated after 50 seconds. After 150 seconds, $\tilde{m}$ was saturated at the lower limit that was specified for the safety. The reason of this nonconvergence is that the update law (31) cannot guarantee to stop the update of the parameters since the law was designed so as to make the tracking error be zero. This practical issue can be avoided by introducing a dead-zone against small error against the update law.

Finally, transitions of the identified parameters of the operator's control model, $T, K_{p}$, and $K_{d}$, are shown in Figures 14,15 , and 16 , respectively. Identified parameter varies during till 30 trials. Transitions of their moving averages are comparatively flat at period of 30-80 trials except rapid change due to large outlier in 53rd trial. Although it is difficult to find tendency of change of the identified characteristics, it was confirmed that their moving averages of parameters $T, K_{p}$, and $K_{d}$ are almost constant after 60 trials of when the tracking error keeps small in Figures 11 and 12. Their constant values do not differ much from their initial values which are ones before the activation of the adaptive control law. In short, it can be considered that total performance was increased by changing the machine side mainly without imposition of large change in human side. This supposition is not authentic since it is not demonstrated by statistical analysis with sufficient number of participants. These are future work.

Results of the experiment, however, showed that the proposed assistive control approach works well, and it can be said that the total performance of whole human-machine system can be enhanced by changing the dynamics of the machine itself.

\section{Conclusion}

For a force-feedback haptic interface system, an adaptive impedance assistive control to enhance the manipulation performance was proposed. The strategy consists of an identification of an operator's control characteristics and an adaptive online tuning of the dynamic property of the machine. The tuning is executed by changing impedance parameters of a virtual internal model for the machine. The adaptive law of the tuning was derived by utilizing a Lyapunov stability concept. Using a haptic 2-DOF interface device, it was demonstrated that proposed adaptive impedance assistive control worked effectively. The tuning law, however, cannot guarantee a convergence to a steady state without reaching to the safety limit yet since the 
presented algorithm was designed by only focusing on an enhancement of the performance of human manipulation without consideration of the machine limit such as an input saturation and frequency bandwidth. This practical issue will be resolved by introducing a tradeoff computation between a performance and requirement of the machine side such as an energy consumption. In the present study, however, a basic strategy for the design of human assistive system could be shown; hence, we would like to treat such practical issues in future.

\section{Acknowledgments}

This work is supported by the Grant-in-Aid for 21st Century (Center of Excellence) COE Program in Ministry of Education, Culture, Sports, Science and Technology. Preparation of the experimental system and the experiment were supported by Keiichi Kurihara and other participants who embraced the authors requests kindly. The authors are grateful to all of them for supporting this work.

\section{References}

[1] T. A. McMahon, "Mechanics of locomotion," International Journal of Robotics Research, vol. 3, no. 2, pp. 4-28, 1984.

[2] A. B. Joaquin and H. Herr, "Adaptive control of a variableimpedance ankle-foot orthosis to assist drop-foot gait," IEEE Transactions on Neural Systems and Rehabilitation Engineering, vol. 12, no. 1, pp. 24-31, 2004.

[3] Y. Yamada, H. Konosu, T. Morizono, and Y. Umetani, "Proposal of skill-assist: a system of assisting human workers by reflecting their skills in positioning tasks," in Proceedings of the IEEE International Conference on Systems, Man, and Cybernetics, vol. 4, pp. 11-16, October 1999.

[4] H. Konosu, I. Araki, and Y. Yamada, "Practical Development of Skill- Assist," Journal of The Robotics Society of Japan, vol. 22, no. 4, pp. 508-514, 2004 (Japanese).

[5] M. Uemura, K. Kanaoka, and S. Kawamura, "Power assist system for sinusoidal motion by passive element and impedance control," in Proceedings of the IEEE International Conference on Robotics and Automation (ICRA '06), pp. 3935-3940, Orlando, Fla, USA, May 2006.

[6] V. Duchaine and C. M. Gosselin, "General model of humanrobot cooperation using a novel velocity based variable impedance control," in Proceedings of the 2nd Joint EuroHaptics Conference and Symposium on Haptic Interfaces for Virtual Environment and Teleoperator Systemsv (WHC '07), pp. 445451, March 2007.

[7] S. Suzuki, K. Kurihara, K. Furuta, F. Harashima, and Y. Pan, "Variable dynamic assist control on haptic system for human adaptive mechatronics," in Proceedings of the 44th IEEE Conference on Decision and Control, and the European Control Conference (CDC-ECC '05), pp. 4596-4601, Seville, Spain, December 2005.

[8] A. Tustin, "The Nature of the Operator's Response in Manual Control and its Implications for Controller Design," Journal of the Institution of Electrical Engineers, vol. 94, no. 2A, pp. 190202, 1947.

[9] J. R. Ragazzini, "Engineering aspects of the human being as a servo-mechanism," in Proceedings of the Meeting of the American Psychological Association, 1948.
[10] S. Baron, D. L. Kleinman, and W. H. Levison, "An optimal control model of human response part II: prediction of human performance in a complex task," Automatica, vol. 6, no. 3, pp. 371-383, 1970.

[11] D. M. Wolpert and M. Kawato, "Multiple paired forward and inverse models for motor control," Neural Networks, vol. 11, no. 7-8, pp. 1317-1329, 1998.

[12] M. Kawato, "Internal models for motor control and trajectory planning," Motor Systems, vol. 9, no. 6, pp. 718-727, 1999.

[13] R. C. Miall, D. J. Weir, D. M. Wolpert, and J. F. Stein, "Is the cerebellum a smith predictor?” Journal of Motor Behavior, vol. 25, no. 3, pp. 203-216, 1993.

[14] K. Kosuge, K. Furuta, and T. Yokoyama, "Virtual model following control of robot arms," IEEE Robotics and Automation, pp. 1549-1554, 1987.

[15] D. L. Kleinman, S. Baron, and W. H. Levison, "An optimal control model of human response part I: theory and validation," Automatica, vol. 6, no. 3, pp. 357-369, 1970.

[16] K. Furuta, Y. Kado, S. Shiratori, and S. Suzuki, "Assisting control for pendulum-like juggling in human adaptive mechatronics," Journal of Systems and Control Engineering IMechE, vol. 225, no. 6, pp. 709-720, 2011.

[17] A. Phatak, H. Weinert, I. Segall, and C. N. Day, "Identification of a modified optimal control model for the human operator," Automatica, vol. 12, no. 1, pp. 31-41, 1976. 

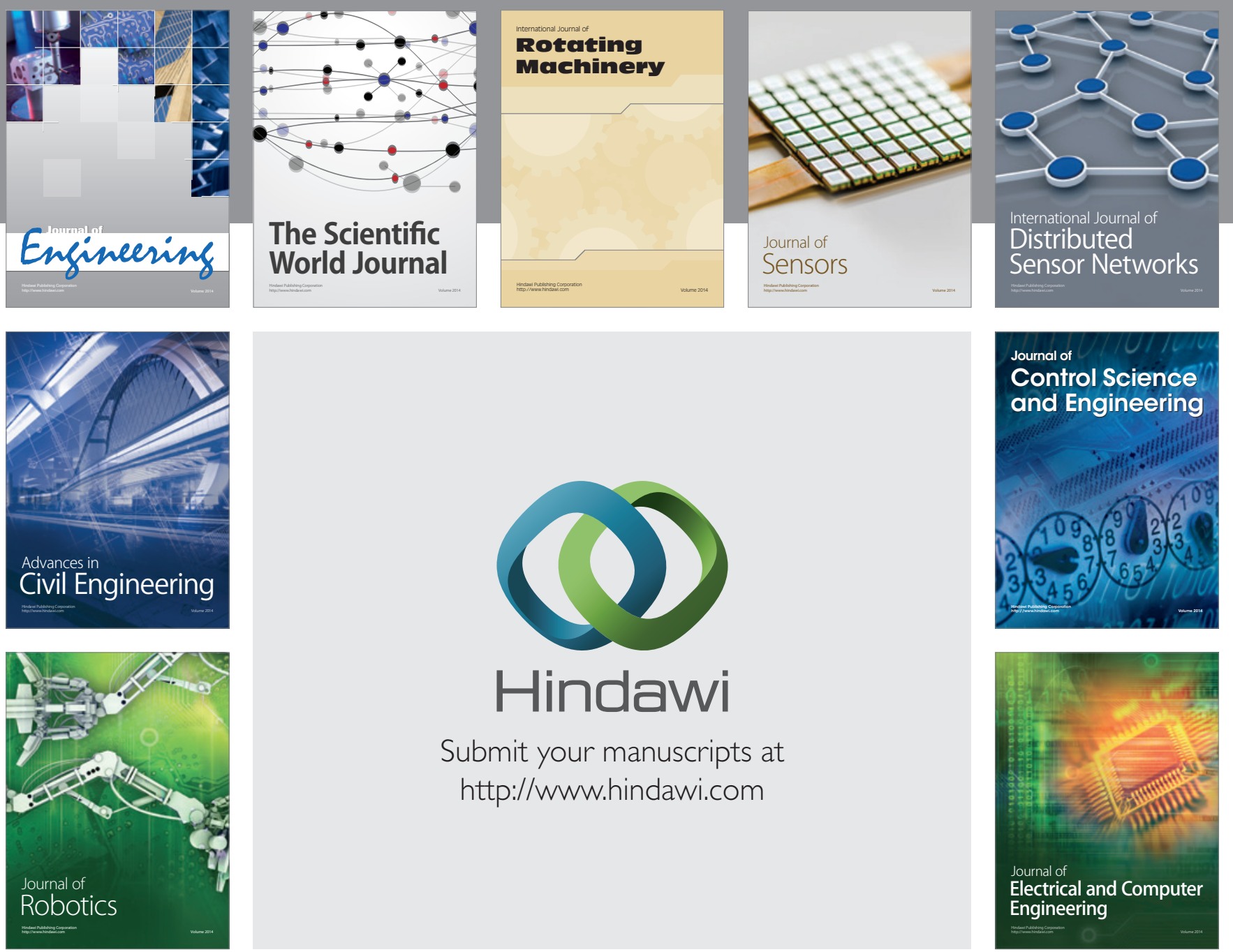

Submit your manuscripts at

http://www.hindawi.com
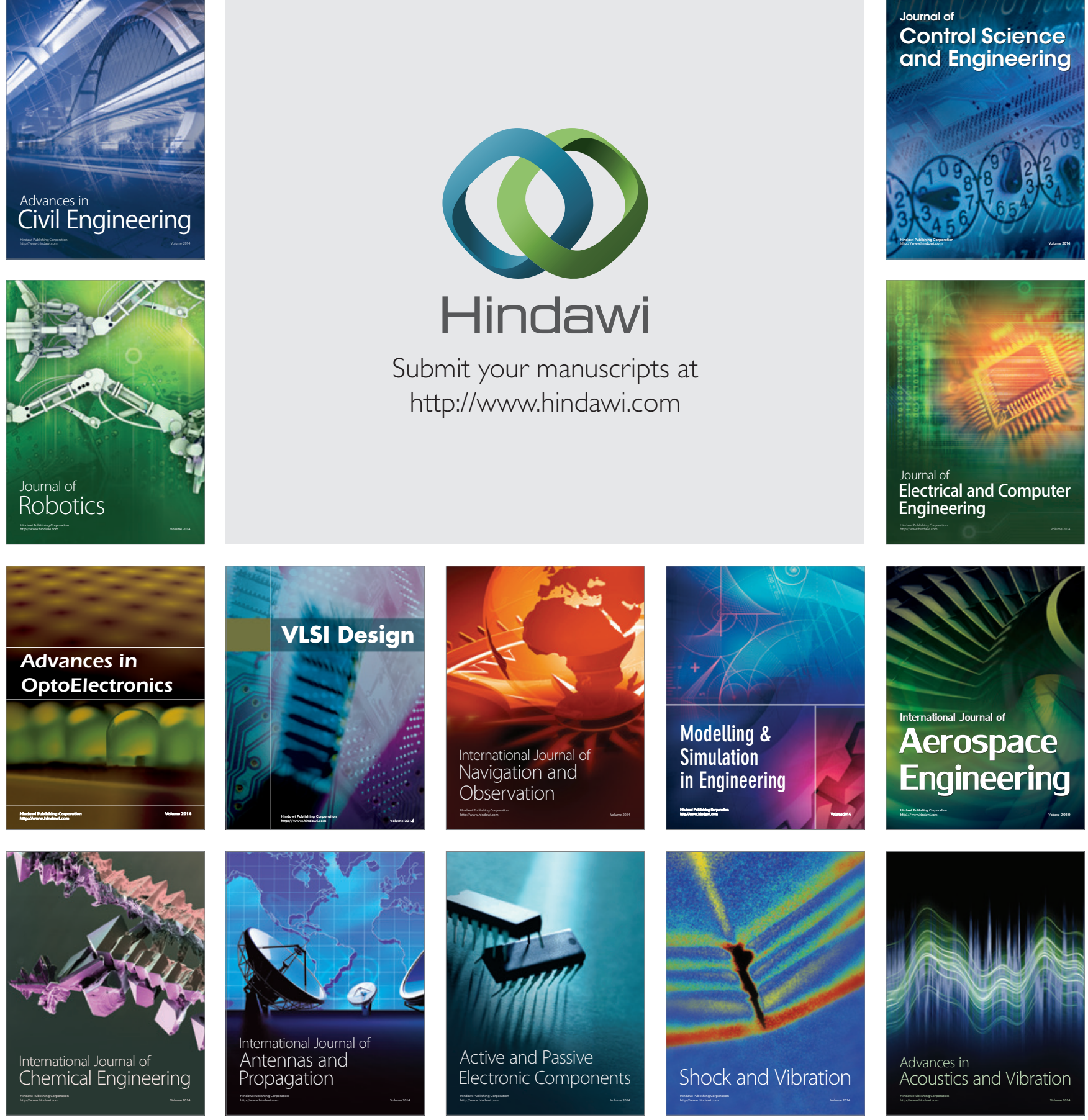